\title{
Naming analog clocks conceptually facilitates naming digital clocks
}

\author{
Marjolein Meeuwissen, ${ }^{\mathrm{a}, *}$ Ardi Roelofs, ${ }^{\mathrm{a}, \mathrm{b}}$ and Willem J. M. Levelt ${ }^{\mathrm{a}}$ \\ a Max Planck Institute for Psycholinguistics, P.O. Box 310, 6500 AH, Nijmegen, The Netherlands \\ ${ }^{\mathrm{b}}$ F.C. Donders Centre for Cognitive Neuroimaging, Nijmegen, The Netherlands
}

Accepted 4 December 2003

Available online 3 February 2004

\begin{abstract}
This study investigates how speakers of Dutch compute and produce relative time expressions. Naming digital clocks (e.g., 2:45, say "quarter to three") requires conceptual operations on the minute and hour information for the correct relative time expression. The interplay of these conceptual operations was investigated using a repetition priming paradigm. Participants named analog clocks (the primes) directly before naming digital clocks (the targets). The targets referred to the hour (e.g., 2:00), half past the hour (e.g., 2:30), or the coming hour (e.g., 2:45). The primes differed from the target in one or two hour and in five or ten minutes. Digital clock naming latencies were shorter with a five- than with a ten-min difference between prime and target, but the difference in hour had no effect. Moreover, the distance in minutes had only an effect for half past the hour and the coming hour, but not for the hour. These findings suggest that conceptual facilitation occurs when conceptual transformations are shared between prime and target in telling time.

(C) 2003 Elsevier Inc. All rights reserved.
\end{abstract}

Keywords: Language production; Semantic facilitation; Conceptual priming; Naming; Time telling; Digital clock; Analog clock

\section{Introduction}

Over the past few decades, there has been an increased interest in numerical cognition and its relation to natural language. Whereas number comprehension and arithmetic processes have been intensively investigated in chronometric, developmental, neuroimaging, and neuropsychological studies (for reviews, Butterworth, 1999; Dehaene, 1997), the production of spoken numerals has not received much attention. Moreover, of the few studies that have been conducted, most have concentrated on the production of relatively simple numerals like those represented by 1- and 2-digit Arabic numerals (e.g., saying "twenty" in response to 20; cf. Brysbaert, 1995; Fias, Reynvoet, \& Brysbaert, 2001). The production of complex numerical expressions like

\footnotetext{
We are indebted to Kay Bock, Alissa Melinger, Pienie Zwitserlood, the members of the Utterance Encoding project of the Max Planck Institute for Psycholinguistics, and two anonymous reviewers for helpful comments and discussions.

${ }^{*}$ Corresponding author. Fax: +31-024-354-1213.

E-mail address: marjolein.meeuwissen@mpi.nl (M. Meeuwissen).
}

those involved in time telling (e.g., saying "quarter to three" in response to 2:45) has been almost completely ignored. Yet, being able to produce complex numerical expressions is an essential numerical skill of adult speakers. Moreover, speakers regularly produce these complex numerical expressions. Thus, no theory of numerical cognition or speech production is complete without an account of the production of complex numerical expressions. The research reported in the present paper might gain insight into certain aspects of the planning processes that underly the production of complex numerical expressions. In particular, we report an experimental study that examined aspects of time telling by native speakers of Dutch.

In one of the first comprehensive experimental studies (to our knowledge the first study) of clock time naming, Bock, Irwin, Davidson, and Levelt (2003) compared time telling by English and Dutch speakers. Dutch and English are closely related languages, yet they differ in their basic expressions for telling time. Whereas English time expressions only make reference to the hour (e.g., the past hour in "ten past $t w o$ " [2:10] and "twenty past two" [2:20] and the coming hour in "quarter to three" 
[2:45]), Dutch time expressions also make reference to the half hour (the same holds for other Germanic languages like German). This secondary reference point in Dutch operates between the $10 \mathrm{~min}$ before and the $10 \mathrm{~min}$ after the half hour, yielding (in literal translations) "ten after $t w o$ " for 2:10, "ten before half three" for 2:20, and "quarter to three" for 2:45. Time expressions in Dutch and English can be relative or absolute. In relative expressions, the relation between the (half) hourly reference point and the minute is explicitly mentioned (e.g., by past in "ten past two" in English), whereas in absolute expressions it is not (e.g., "two-ten" in English). Furthermore, in relative expressions the reference point changes between the past hour and the coming hour (compare "ten past two" for 2:10 with "quarter to three" for 2:45), whereas in absolute expressions it remains the same (e.g., "two forty-five" for 2:45). Bock et al. (2003) found that American-English speakers strongly favor absolute over relative expressions, whereas Dutch speakers strongly prefer relative over absolute expressions. The preferences did not differ between analog and digital clocks. Moreover, Bock et al. had English and Dutch speakers tell the time from analog and digital clocks while recording the speakers' eye movements and naming latencies. The data revealed a tight link between the way clock displays are visually inspected by a speaker and the subsequent production of the time expressions. It seems that within $300 \mathrm{~ms}$ after the presentation onset of a clock, a speaker constructs a conceptual representation of the time information to be expressed, which is used to incrementally generate the corresponding spoken utterance.

Recently, we (Meeuwissen, Roelofs, \& Levelt, in press) developed a working model for the conceptual operations involved in relative time telling in Dutch within the framework of the theory of lexical access in speech production advanced by Levelt, Roelofs, and Meyer (1999; Roelofs, 1992, 1997, 2003). This theory holds that the conceptually driven planning of spoken utterances is a staged process, traversing from conceptual preparation to the initiation of articulation. Three planning levels are distinguished: conceptual preparation, lemma retrieval, and form encoding. Conceptual preparation involves the construction of a conceptual representation (in terms of lexical concepts and their relationships) for the information to be verbally expressed. Lemma retrieval involves the retrieval of memory representations of the syntactic properties of the lexical items expressing the conceptual information. After having selected lemmas, the form of the utterance is encoded, which includes access to the required morphological, phonological, and phonetic information. Telling the time from an analog clock in Dutch (e.g., "kwart voor drie," English "quarter to three") involves determining the hours and minutes from the hands of the clock and conceptually determining the reference point (past hour, half past the hour, or coming hour) and the distance from the reference point in minutes. Next, the corresponding lemmas need to be retrieved (e.g., kwart, English quarter; voor, English to; and drie, English three) and syntactically ordered. Finally, the corresponding morphemes (e.g., <kwart $>$ ) and phonemes $(/ \mathrm{k} /, / \mathrm{w} /$, etc.) need to be retrieved, the phonemes should be syllabified, and the corresponding motor programs need to be recovered. Naming digital clock times (e.g., 2:45) minimally involves determining the hours and minutes from the digits in the input, conceptually determining the reference point (for 2:45 this is the coming hour, i.e., three) and the distance from the reference point in minutes (i.e., 15), retrieving the corresponding lemmas (the lemma kwart for 15 and the lemmas of voor and drie) and syntactically ordering them, retrieving the corresponding morphemes and phonemes, syllabifying the phonemes, and recovering the corresponding motor programs (Fig. 1).

We reported evidence for the engagement of these planning levels in the production of clock times (Meeuwissen et al., in press). In two experiments conducted in Dutch, we compared the production of clock times and house numbers in response to three-digit Arabic numerals as stimuli, which ranged from 200 to 955 in steps of 5. For example, the participants said "op driehonderdvijfenveertig" ("at three-hundred forty-five") to 345 and "om kwart voor vier" ("at quarter to four") to 3:45. Multiple regression analyses showed that the naming latencies for the house numbers were mostly determined by morphophonological factors such as the number of phonemes and morphemes and by morpheme frequency. In contrast, the latencies of the clock time naming revealed a strong additional conceptual involvement. The latency patterns here were best explained by assuming a number of conceptual operations applied to the input (e.g., 3:45 should

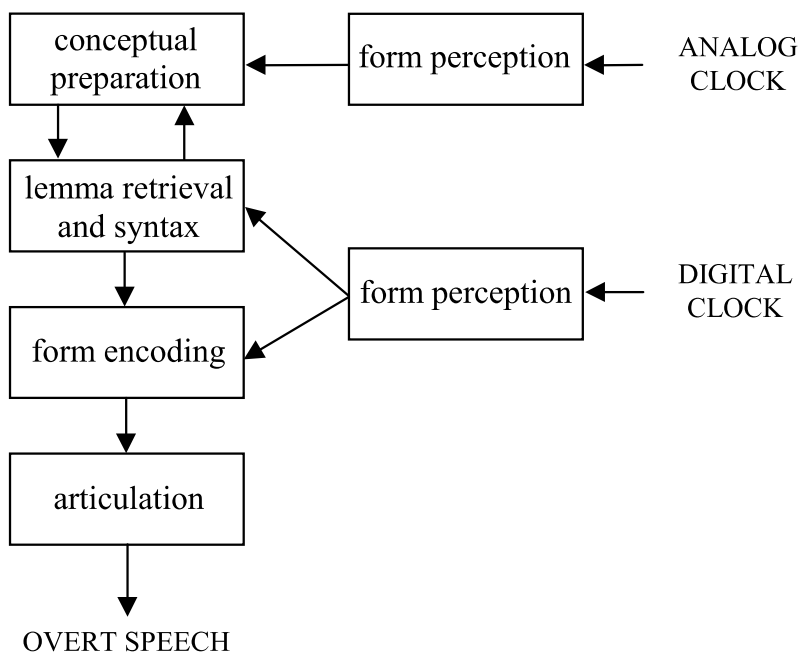

Fig. 1. Planning levels in digital and analog clock time naming following Meeuwissen, Roelofs, and Levelt (in press). 
be conceptually transformed into quarter to four). A second study showed that when these conceptual operations were made unnecessary by presenting alphabetically written numerals as stimuli (e.g., DRIEHONDERDVIJF ENVEERTIG, English "three-hundred forty-five;" KWART VOOR VIER, English "quarter to four"), only morphophonological factors determined the production latencies in both response modes. These results indicate that different planning levels are engaged in spoken numeral production depending on the response mode and perceptual input format.

As concerns the conceptual preparation required for digital clock time naming, the differences in reference point (past hour, half past the hour, and coming hour) were reflected in the naming latencies. Utterances referring to the past hour were produced faster than utterances referring to the half hour and the coming hour. Furthermore, the differences in minutes were also reflected in the naming latencies. Utterances mentioning a $10 \mathrm{~min}$ distance from a reference point were produced slower than utterances mentioning a 5 min distance, and these were produced slower than utterances that expressed a zero distance.

We explained these findings on the conceptual preparation of clock time expressions in terms of a procedural semantics for clock time naming. In constructing a conceptual representation for a clock time on the basis of digital input, a speaker of Dutch has to determine the reference point and the distance in minutes. For a given 3-digit input $\mathrm{X}: \mathrm{YZ}$, a procedure is assumed that, as a first step, determines the reference point. If $\mathrm{YZ}$ is smaller than 20, the reference point is $x$, if it falls between 20 and 40 , the reference point is half $(x+1)$, and if it is larger than 40 , the reference point is $(x+1)$. If adding an hour takes time, utterances referring to the past hour (" $x$ ") should be produced faster than those referring to the coming hour (" $x+1$ ") and half past the hour ("half $(x+1)$ "), as we empirically observed. Our data suggested that, overall, specifying a 5 min distance takes less time than specifying a ten minute distance. However, the effect of the minutes (five versus ten) depended on the reference point (past hour, half past the hour, and coming hour). The difference between specifying five and ten minutes was around $25 \mathrm{~ms}$ for half past the hour and the coming hour, but it was only $5 \mathrm{~ms}$ for the past hour. This was explained by referring to the fact that specifying the minutes relative to the half hour and coming hour requires a numerical transformation of the input (e.g., the 20 in 2:20 has to be transformed into ten minutes for "tien voor half drie," in English literally "ten before half three" and the 25 in 2:25 into 5 min for "vijf voor half drie", in English literally "five before half three"), whereas such transformations are not required for the past hour (e.g., the 05 in 2:05 remains 5 min for "vijf over twee", in English "five past two," and the 10 in 2:10 remains $10 \mathrm{~min}$ for "tien over twee," in English "ten past two"). In summary, the findings for the clock times were taken to support a procedural semantics in which reference point and distance in minutes information from the digital input had to be conceptually transformed in order to produce the correct relative time expression.

The present study investigated the interplay of these conceptual operations using a repetition priming paradigm (cf. Bock, 1986; Bock \& Griffin, 2000; Bock \& Loebell, 1990; Wheeldon \& Monsell, 1994). We investigated whether naming an analog clock (the prime) before naming a digital clock (the target) has an effect on target production latencies. Participants told the time from an analog clock directly before telling the time from a digital clock. The digital target clock times referred to the hour (e.g., 2:00), half past the hour (e.g., 2:30), or the coming hour (e.g., 2:45). The analog clock primes differed from the targets in one or two hours and in five or ten minutes. As a control priming condition, we considered to include an analog clock without any time information or an analog clock that displays the same time as the target. However, there are several problems with these candidate control conditions. When the prime contains no time information, the task for prime and target is different (i.e., all primes would require an oral response except for the control condition). And there would be full conceptual and form overlap when prime and target would display the same time. Since we were not interested in determining whether priming produces "absolute" facilitation or interference but only in assessing whether conceptual priming of minute and hour information occurs, we decided to include no "neutral" control condition.

In our experiment, participants named an analog clock as prime before naming a digital clock. Other prime-target combinations are of course possible, such as an analog clock as prime for an analog clock or a digital clock as prime for a digital clock. However, with such prime-target combinations there would be visual overlap between prime and target, making it difficult to assess the conceptual contribution. So, in order to pick up the "true" conceptual contribution, it is important that primes and targets share no visual overlap. This is the case in our study.

If aspects of conceptual transformations (i.e., procedure applications or memory retrievals) are shared between naming analog and digital clocks, repetition priming effects should be obtained. Whereas determining the reference point from analog clocks is based on the spatial position of the big hand of the clocks, determining the reference point from digital clocks is based on the last two digits specifying the minutes, as explained earlier. If the number of minutes specified by the big hand or the last two digits is smaller than 20 , the reference point is $x$, if it falls between 20 and 40, the reference point is half $(x+1)$, and if it is larger than 40 , 
the reference point is $(x+1)$. For analog clocks, the value of $x$ is determined by the spatial position of the small hand and for digital clocks the value is determined by the first, leftmost digit. Given that all these operations differ between display formats (using the spatial position of the hands versus using digital information), one expects that priming the reference points has no effect. In contrast, determining the distance in minutes relative to the reference point seems to have certain aspects in common between naming digital and analog clocks. In particular, if the minute information is encoded into the same conceptual format for digital and analog clocks, determining the minutes relative to the reference point can be performed in the same way for both clock types. Consequently, one expects repetition priming for a difference in minutes between prime and target.

\section{Method}

\subsection{Participants}

Eighteen speakers participated in the experiment. They were undergraduate students of Nijmegen University, native speakers of Dutch, and they had normal or corrected-to-normal vision. They were paid for their participation.

\subsection{Materials and design}

Three types of digital clock times were used as targets, henceforth referred to as type: the hour (2:00, $3: 00, \ldots, 9: 00)$, half past the hour $(2: 30,3: 30, \ldots, 9: 30)$, and quarter to the coming hour $(2: 45,3: 45, \ldots, 9: 45)$. Of each target type, there were 8 instances (e.g., for the hour, the instances were 2:00, 3:00, 4:00, 5:00, 6:00, 7:00, 8:00, and 9:00). The primes consisted of analog clocks with big and small hands but not containing any numbers or interval marks. The primes depicted times from the 12-h period between 12:00 and 11:55 in five-minute intervals. Primes differed in time from the targets both on the hour (one versus two hours) and minute (five versus ten minutes) dimension. Primes depicted times that were always later than targets. For example, for the target 2:00 (say "twee uur," English "two o'clock") analog clock primes would elicit utterances like "vijf over drie" (English "five past three"), "tien over drie" (English "ten past three"), "vijf over vier" (English "five past four"), or "tien over vier" (English "ten past four"). A similar approach was taken for the other two target types, with primes either being one hour and five minutes, one hour and ten minutes, two hours and five minutes, or two hours and ten minutes later than the targets.

Table 1 lists example utterances for each of the four prime conditions and each of the three target types. Note that for the target type of quarter to the next hour (e.g., 2:45), analog clock primes would elicit utterances like "tien voor vier" (English "ten before four") resulting in a one-hour and five-minute difference between prime and target, and "vijf voor vier" (English "five to four") resulting in a one-hour and ten-minute distance between prime and target. Whereas the prime utterances express a five or ten minutes distance from the coming hour, the distance from the target reference point (i.e., quarter to the coming hour) is the reverse. For example, the distance between the prime "vijf voor vier" (English "five to four") and the target "kwart voor vier" ("quarter to four") is ten rather than five minutes. We expected that the distance from the target expression is critical (e.g., ten minutes), not the number of minutes specified in the prime expression (e.g., five).

Table 1

Example utterances for each prime condition and each target type, with literal English translations between parentheses. Primes were either $1 \mathrm{~h}$ and $5 \mathrm{~min}, 1 \mathrm{~h}$ and $10 \mathrm{~min}, 2 \mathrm{~h}$ and $5 \mathrm{~min}$, and $2 \mathrm{~h}$ and $10 \mathrm{~min}$ later than the target

\begin{tabular}{llll}
\hline Prime condition (distance) & Prime utterance (from analog display) & Target utterance (from digital display) \\
\hline$\frac{\text { Hour }}{1}$ & Minute & "vijf over vier" (five past four) & Full hour \\
1 & 5 & "tien over vier" (ten past four) & "drie uur" (three o'clock) \\
2 & 10 & "vijf over vijf" (five past five) & "drie uur" (three o'clock) \\
2 & 10 & "tien over vijf" (ten past five) & "drie uur" (three o'clock) \\
& & "drie uur" (three o'clock) \\
1 & 5 & "vijf over half vier" (five past half four) & Half past the hour \\
1 & 10 & "tien over half vier" (ten past half four) & "half drie" (half three) \\
2 & 5 & "vijf over half vijf" (five past half five) & "half drie" (half three) \\
2 & 10 & "tien over half vijf" (ten past half five) & "half drie" (half three) \\
& 5 & "tien voor vier" (ten before four) & Quarter to the coming hour \\
1 & 10 & "vijf voor vier" (five before four) & "kwart voor drie" (quarter to three) \\
1 & 5 & "tien voor vijf" (ten before five) & "kwart voor drie" (quarter to three) \\
2 & 10 & "vijf voor vijf" (five before five) & "kwart voor drie" (quarter to three) \\
2 & &
\end{tabular}


We decided to restrict the experimental targets to the hour, half past the hour, and quarter to the coming hour to prevent a problem associated with the priming of relative clock times in Dutch. Consider, for example, the analog clock for "tien voor half vier" (English "ten before half four") as a prime for the target 3:15. Although the difference between prime and target is only five minutes, the reference point of the prime is simultanously changed by one hour. To avoid such complications, we used only the hour, half past the hour, and quarter to the coming hour as targets. However, to avoid that participants became aware of the prime-target relations, a great number of filler primes and targets were included in the experiment. Filler trials had as targets the full hour, quarter past the hour, half past the hour, and quarter to the coming hour, which were combined with filler primes that varied only on the hour or only on the minute dimension with the targets (but not on both dimensions). This introduced phonological overlap between prime and target (e.g., "drie uur," English "three o'clock" served as prime for "twee uur," English "two o'clock," or "vijf over drie," English "five past three" served as prime for "vijf over twee," English "five past two"). Furthermore, the distance between filler primes and targets could be more than one or two hours and more than five or ten minutes (e.g., "tien voor half vier," English "ten before half hour" served as prime for "twee uur," English "two o'clock"). We controlled for possible voicekey artifacts by instructing participants to start each response with the same word, om (English at) in both analog and digital clock time naming. In this way, the responses in all conditions started with the same phoneme.

The experimental trials were preceded by a block of practice trials containing all the analog clock faces in a random fashion. After the practice block, the experimental analog primes and digital targets were presented in a random fashion, each target occurring once in each of the four prime conditions (one hour and five minutes; one hour and ten minutes; two hours and five minutes; and two hours and ten minutes), yielding 96 trials in total ( 3 target types $\times 8$ instances $\times 4$ prime conditions). In addition, there were 342 filler trials.

\subsection{Procedure}

The clock faces were displayed on a ViewSonic 17PS screen. The experiment was run under the Nijmegen experiment setup (NESU). Participants' utterances were recorded over a Sennheiser ME400 microphone to a Sony DTC55 digital audio tape recorder for later transcription. An electronic voice key measured the naming latencies. Participants were tested individually. They were seated in a dimly lit, soundproof cabin in front of the computer monitor. The distance between participant and screen was approximately $50 \mathrm{~cm}$. Preceding the experiment, participants were provided with a written instruction stating that the clocks (both analog and digital) had to be named in a relative way, with some illustrative examples. Furthermore, they were asked to respond in a fluent manner. The structure of a trial was as follows: First, the participant saw a warning signal (an asterisk) for $500 \mathrm{~ms}$, directly followed by the display of an analog clock (the prime) in the middle of the screen for four seconds. This long presentation time for the analog clocks was chosen based on the study by Bock et al. (2003) and a small pilot study. Bock et al. observed that speakers of Dutch start uttering a relative clock time expression from an analog clock on average $1300 \mathrm{~ms}$ after display onset. In our pilot study, we observed that participants were able to complete the naming of analog clocks within four seconds. Thus, by displaying the analog clock for four seconds, the participants should be able to complete the naming of the analog clocks before the digital clocks are presented. After displaying an analog clock (the prime), a digital clock (the target) was displayed in the middle of the analog clock for one second. Before the next trial started, the screen went blank for one second. Stimuli were presented in black on a white background. The total duration of the trial was $6.5 \mathrm{~s}$. An experimental session lasted about $50 \mathrm{~min}$.

\subsection{Analyses}

A trial was considered invalid when it included a mispronunciation, when a wrong response was produced, or when the voice key was triggered incorrectly. Invalid trials were excluded from the statistical analyses of the latencies. The naming latencies and errors for the digital clocks were submitted to by-participant and byitem analyses of variance (ANOVAs). Minute and hour were tested within participants and within items. Target was tested within participants but between items.

\section{Results}

Table 2 gives the mean naming latencies, the standard deviations, and the error percentages for the target by hour by minute cells. Fig. 2 shows the mean naming latencies for each prime condition.

\subsection{Naming latencies}

Naming latencies differed depending on target, $F_{1}(2$, $34)=37.55, M S E=7996, p<.001, F_{2}(2,21)=72.57$, $M S E=1758, p<.001$. Participants were fastest in producing full hours (on average $521 \mathrm{~ms}$ ), followed by the half hours (on average $621 \mathrm{~ms}$ ) and quarter to the coming hours (on average $636 \mathrm{~ms}$ ). This replicates Meeuwissen et al. (in press), who tested these three target types 
Table 2

Mean production latencies ( $M$, in milliseconds), standard deviations $(S D)$, and error percentages $(E \%)$ per target type and prime type

\begin{tabular}{|c|c|c|c|c|c|c|c|}
\hline \multirow[t]{4}{*}{ Target } & \multicolumn{7}{|l|}{ Prime } \\
\hline & \multirow[t]{3}{*}{ Hour } & \multicolumn{6}{|c|}{ Minute } \\
\hline & & \multicolumn{3}{|l|}{5} & \multicolumn{3}{|l|}{10} \\
\hline & & $M$ & $S D$ & $E^{\circ} \%$ & $M$ & $S D$ & $E \%$ \\
\hline \multirow[t]{3}{*}{ Full hour } & 1 & 529 & 103 & 3.5 & 510 & 87 & 4.9 \\
\hline & 2 & 521 & 89 & 4.2 & 522 & 101 & 3.5 \\
\hline & Total & 525 & 96 & 3.9 & 516 & 94 & 4.2 \\
\hline \multirow[t]{3}{*}{ Half past the hour } & 1 & 606 & 157 & 2.8 & 627 & 153 & 4.9 \\
\hline & 2 & 608 & 142 & 6.3 & 641 & 171 & 6.9 \\
\hline & Total & 607 & 150 & 4.6 & 634 & 162 & 5.9 \\
\hline \multirow[t]{3}{*}{ Quarter to coming hour } & 1 & 617 & 158 & 7.6 & 665 & 170 & 9.7 \\
\hline & 2 & 622 & 147 & 4.9 & 643 & 182 & 10.4 \\
\hline & Total & 620 & 152 & 6.3 & 654 & 176 & 10.1 \\
\hline
\end{tabular}

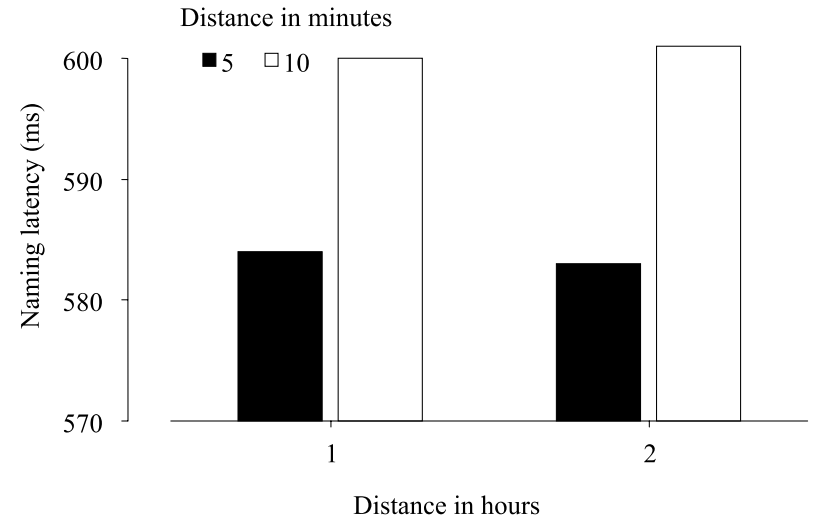

Fig. 2. Target naming latencies in milliseconds collapsed across target type as a function of distance in hours and minutes between prime and target.

together with other targets ranging from 2:00 to 9:55 in steps of $5 \mathrm{~min}$. Priming the hour had no effect, $F_{1}(1,17)<1, \quad M S E=1259, \quad p>.95, \quad F_{2}(1,21)<1$, $M S E=1116, p>.92$. However, priming the minutes affected the latencies, $F_{1}(1,17)=6.67, M S E=2404$, $p<.02, F_{2}(1,21)=6.97, M S E=1078, p<.02$ (mean naming latencies were $584 \mathrm{~ms}$ for a five-minute distance and $600 \mathrm{~ms}$ for a ten-minute distance from the target). There was no interaction between hour and minute, $F_{1}(1,17)<1, \quad M S E=2191, \quad p>.95, \quad F_{2}(1,21)<1$, $M S E=1331, p>.95$. The effect of the distance in minutes varied with target, $F_{1}(2,34)=5.40, M S E=1733, p<.01$, $F_{2}(2,21)=3.98, M S E=1078, p<.04$. The effect of minute was only obtained for the targets referring to the half hour $(27 \mathrm{~ms})$ and quarter to the next hour $(34 \mathrm{~ms})$, whereas the targets referring to the full hour showed no effect $(-9 \mathrm{~ms})$. There were no interactions of target and hour, $F_{1}(2,34)=1.68, M S E=945, p>.20, F_{2}(2,21)<1$, $M S E=1116, p>.66$, and of target, hour and minute, $F_{1}(2,34)=2.94, \quad M S E=1360, p>.06, \quad F_{2}(2,21)<1$, $M S E=1331, p>.44$.

\subsection{Errors}

The analysis of the errors yielded a main effect of target, $F_{1}(2,34)=4.33, p<.03, F_{2}(2,21)=4.25, p<.03$. The number of errors was lowest when the target made reference to the full hour $(4.0 \%)$, followed by the half hour $(5.2 \%)$, and the quarter to the coming hour $(8.2 \%)$. As indicated by Table 2, most errors were made in the slowest conditions, so there is no evidence for a speed-accuracy tradeoff. There were no main effects of hour, $F_{1}(1,17)<1, p>.61, F_{2}(1,21)<1, p>.80$, and minute, $F_{1}(1,17)=2.63, p>.10, \quad F_{2}(1,21)=2.07, p>.16$. Moreover, there were no two-way or three-way interactions (most $F \mathrm{~s}<1$ ).

\section{General discussion}

Earlier research suggested that naming digital clocks involves conceptual operations carried out on the hour and minute information from the digital input (Meeuwissen et al., in press). The present study investigated the interplay of these conceptual operations using a repetition priming paradigm. Dutch participants told the time from an analog clock (the prime) directly before telling the time from a digital clock (the target). The target clock times referred to the hour (e.g., 2:00), half past the hour (e.g., 2:30), or the coming hour (e.g., 2:45). The prime clocks differed from the target in one or two hours and in five or ten minutes. Digital naming latencies were shorter with a five-minute difference than with a ten-minute difference, whereas the difference in hour had no effect, and there was also no interaction between minute and hour. The distance in minutes had only an effect when the utterance made reference to half past the hour or the coming hour, but not when reference was made to the hour.

The absence of a repetition priming effect for the hours suggests that determining the reference point ( $x$, half $(x+1)$, and $x+1)$ and its value (e.g., $x$ takes the 
value 3) from the hands of an analog clock does not help to determine the reference point and its value from the digits in a digital clock. This suggests that determining the reference point and its value are different operations for analog and digital clocks. The presence of a repetition priming effect for the minutes suggests that determining the distance from the reference point in minutes for an analog clock helps to determine the distance from the reference point in minutes for a digital clock. This suggests that, once the reference point and the minutes are known, determining the minutes relative to the reference point is the same operation for analog and digital clocks. Moreover, whereas specifying the minutes relative to the half hour and the coming hour requires numerical transformations of the minute information provided by the digital input (e.g., the 20 in 2:20 has to be transformed into ten minutes, the 25 in 2:25 into five minutes, and the 30 in 2:30 into zero minutes) and the hands of an analog clock face (e.g., the thirty minutes determined for half past two should be transformed into zero minutes relative to the half-past reference point), such transformations are not required for the full hour (e.g., the 00 in 2:00 remains zero minutes, the 05 in 2:05 remains five minutes, and the 10 in 2:10 remains ten minutes). This may explain why an effect of minutes is obtained for half past the hour and the coming hour, but not for the hour. Moreover, it may explain why an effect of minutes is obtained both in specifying the conceptual representation for the utterance (Meeuwissen et al., in press) and also in priming this specification.

Since speakers responded fastest to the full hours, one may wonder whether the absence of a priming effect for the full hour is a floor effect. This seems unlikely, though, upon inspection of Table 2. It appears that a ten-minute difference between prime and target yielded numerically smaller latencies $(516 \mathrm{~ms})$ than a five-minute difference $(525 \mathrm{~ms})$. Thus, the direction of the priming effect is different for the full hour than for half past the hour and the coming hour, which suggests that the priming effect is differently mediated for the full hour than for the other target types, as we suggested.

We examined time telling by speakers of Dutch. Given that the systems for telling time differ between languages, one may wonder whether the present results are specific for the Dutch language. As we explained, whereas English has only an hourly reference point, Dutch has both hourly and half-hourly reference points. In our study, conceptual priming was obtained for both the hourly reference point (i.e., the coming hour) and the half-hourly reference point. Given that both Dutch and English make reference to the coming hour, we expect that the conceptual priming we obtained will also be obtained for English. This may be tested in future research.

To conclude, naming analog clocks conceptually facilitates naming digital clocks. The facilitation is observed for the minutes but not for the hours. This suggests that determining the reference point is accomplished in a different way, whereas determining the minutes relative to the reference point has certain aspects in common for analog and digital clocks. This account predicts that an effect of distance in hour should be obtained when digital clocks are used as primes for naming digital clocks and analog clocks are used as primes for naming analog clocks. The current study has shown that priming is possible without visual overlap between prime and target, which opens the way for testing these predictions in future research.

\section{References}

Bock, J. K. (1986). Syntactic persistence in language production. Cognitive Psychology, 18, 355-387.

Bock, J. K., \& Loebell, H. (1990). Framing sentences. Cognition, 35, 139.

Bock, J. K., \& Griffin, Z. M. (2000). The persistence of structural priming: Transient activation or implicit learning? Journal of Experimental Psychology: General, 129, 177-192.

Bock, K., Irwin, D. E., Davidson, D. J., \& Levelt, W. J. M. (2003). Minding the clock. Journal of Memory and Language, 48, 653-685.

Brysbaert, M. (1995). Arabic number reading: On the nature of the numerical scale and the origin of phonological recoding. Journal of Experimental Psychology: General, 124, 434-452.

Butterworth, B. (1999). The mathematical brain. London: Macmillan.

Dehaene, S. (1997). The number sense: How the mind creates mathematics. New York: Oxford University Press.

Fias, W., Reynvoet, B., \& Brysbaert, M. (2001). Are Arabic numerals processed as pictures in a Stroop interference task? Psychological Research, 65, 242-249.

Levelt, W. J. M., Roelofs, A., \& Meyer, A. S. (1999). A theory of lexical access in speech production. Behavioral and Brain Sciences, $22,1-38$.

Meeuwissen, M., Roelofs, A., \& Levelt, W. J. M. (in press). Planning levels in naming and reading complex numerals. Memory and Cognition.

Roelofs, A. (1992). A spreading-activation theory of lemma retrieval in speaking. Cognition, 42, 107-142.

Roelofs, A. (1997). The WEAVER model of word-form encoding in speech production. Cognition, 64, 249-284.

Roelofs, A. (2003). Goal-referenced selection of verbal action: Modeling attentional control in the Stroop task. Psychological Review, $110,88-125$.

Wheeldon, L. R., \& Monsell, S. (1994). Inhibition of spoken word production by priming a semantic competitor. Journal of Memory and Language, 33, 332-356. 